\title{
Sistema de observación para la evaluación técnica en la danza clásica: ejercicio del plié
}

\section{Observation system for technical evaluation in classical dance: plié exercise}

\section{Sistema de observação para avaliação técnica em dança clásica: exercício \\ plié}

\author{
Sánchez, $\mathrm{M}^{1}$., Morillo-Baro, J. P²., Quiñones, $\mathrm{Y}^{3}$., Morales- Sánchez, V., Hernández-Mendo, A. ${ }^{5}$ \\ Facultad de Psicología, Universidad de Málaga 1,2,4,5; Facultad de Cultura Física, Universidad de Pinar \\ del Río, $\mathrm{Cuba}^{3}$
}

\begin{abstract}
RESUMEN
La danza clásica es una disciplina rigurosa, técnica, estilística, cultural y artística que requiere el estudio de las acciones y habilidades motrices. El estudio presenta una herramienta de observación ad hoc compuesta por un sistema mixto de formato de campo y un sistema de categorías exhaustivas y mutuamente excluyentes (E/ME), con el objetivo de validarla para poder codificar y evaluar un ejercicio de danza clásica, el plié de una barra de ballet. El instrumento está compuesto por 5 criterios y un total de 84 categorías distribuidas de la siguiente forma: 9 para las cuentas musicales, 19 para cabeza/mirada, 33 en tren inferior, 18 en tren superior y 5 en dirección espacial. La muestra fueron 10 bailarines/as, ocho mujeres y dos hombres, todos con un nivel profesional en danza clásica. Se realizó un análisis de Calidad del Dato y un análisis de Generalizabilidad con los programas HOISAN y SAGT v1.0. La fiabilidad de los observadores se llevó a cabo mediante el cálculo de los coeficientes de correlación Pearson, Spearman y Tau b de Kendall; y mediante el índice de concordancia Kappa de Cohen y concordancia canónica de Krippendorf. Las correlaciones estimadas fueron de .99-1.00 para la fiabilidad inter e intraobservador, el índice de Kappa de Cohen se situó entre .99 y 1.00 respectivamente y la concordancia canónica entre $99.6 \%$ y 100\%. Los resultados muestran índices adecuados de correlación, así como excelentes resultados de generalizabilidad con un valor $\mathrm{G}$ relativo y $\mathrm{G}$ absoluto de 1.00 en el acuerdo interobservador e intraobservador, revelando que la herramienta de observación presenta una óptima validez, precisión y fiabilidad.
\end{abstract}

Palabras clave: Metodología Observacional, Análisis de Generalizabilidad, Calidad del Dato, Danza Clásica, Plié.

\begin{abstract}
Classical dance is a rigorous, technical, stylistic, cultural and artistic discipline that requires the study of actions and motor skills. The study presents an ad hoc observation tool composed of a mixed field format system and a system of exhaustive and mutually exclusive categories (E/ME), with the aim of validating it in order to be able to code and
\end{abstract}




\section{Observación de la evaluación técnica en danza clásica}

evaluate a classical dance exercise, the plie of a ballet barre. The instrument is made up of 5 criteria and a total of 84 categories distributed as follows: 9 for musical accounts, 19 for head / gaze, 33 in lower body, 18 in upper body and 5 in spatial direction. The sample was 10 dancers, eight women and two men, all with a professional level in classical dance. A Data Quality analysis and a Generalizability analysis were performed with the HOISAN and SAGT v1.0 programs. The reliability of the observers was carried out by calculating the correlation coefficients Pearson, Spearman and Kendall's Tau b; and using Cohen's Kappa concordance index and Krippendorf's canonical concordance. The estimated correlations were .99-1.00 for inter and intra-observer reliability, Cohen's Kappa index was between .99 and 1.00 respectively, and canonical agreement between $99.6 \%$ and $100 \%$. The results show adequate correlation indices, as well as excellent generalizability results with a relative $G$ and absolute $G$ value of 1.00 in the interobserver and intraobserver agreement, revealing that the observation tool has optimal validity, precision and reliability.

Keywords: Observational Methodology, Generalizability Analysis, Data Quality, Classical Dance, Plié.

\section{RESUMO}

A dança clássica é uma disciplina rigorosa, técnica, estilística, cultural e artística que requer o estudo das ações e habilidades motoras. O estudo apresenta uma ferramenta de observação ad hoc composta por um sistema de formato de campo misto e um sistema de categorias exaustivas e mutuamente exclusivas (E/ME), com o objetivo de validála para poder codificar e avaliar um exercício de dança clássica, a folha de uma barra de balé. O instrumento é composto por 5 critérios e um total de 84 categorias distribuídas da seguinte forma: 9 para contas musicais, 19 para cabeça / olhar, 33 na parte inferior do corpo, 18 na parte superior do corpo e 5 na direção espacial. A amostra foi de 10 bailarinos, sendo oito mulheres e dois homens, todos com nível profissional em dança clássica. Uma análise de qualidade de dados e uma análise de generalização foram realizadas com os programas HOISAN e SAGT v1.0. A confiabilidade dos observadores foi realizada por meio do cálculo dos coeficientes de correlação de Pearson, Spearman e Kendall's Tau b; e usando o índice de concordância Kappa de Cohen e a concordância canônica de Krippendorf. As correlações estimadas foram de 0,99 a 1,00 para confiabilidade inter e intraobservador, o índice Kappa de Cohen ficou entre 0,99 e 1,00, respectivamente, e a concordância canônica entre 99,6\% e 100\%. Os resultados mostram índices de correlação adequados, bem como excelentes resultados de generalizabilidade com $\mathrm{G}$ relativo e valor $\mathrm{G}$ absoluto de 1,00 na concordância interobservador e intraobservador, revelando que o instrumento de observação tem validade, precisão e confiabilidade ótimas.

Palavras chave: Metodologia Observacional, Análise de Generalizabilidade, Qualidade de Dados, Dança Clássica, Plié.

\section{INTRODUCCIÓN}

El ballet, en la forma en que se reconoce hoy, es esencialmente un producto del Renacimiento italiano (Haskell, 1973). La danza clásica es una disciplina que integra una técnica progresiva y rigurosa (Taccone, 2016). La ejecución y la interpretación de la danza clásica o académica precisa de un profundo conocimiento y dominio del cuerpo. Además, implica una extraordinaria exhibición de destreza física (Greene-Hass, 2010).

En los primeros años de estudio, en las clases de danza clásica, se trabaja la correcta colocación del cuerpo, haciendo hincapié en el desarrollo de una base sólida técnica y expresiva. Los cursos profesionales trabajan la perfección y el dominio de los elementos fundamentales de la técnica de la danza académica. Cada combinación en la barra y en el centro debe tener un propósito definido (Greene-Hass, 2010).

La danza clásica profesional exige en los bailarines y bailarinas una alta preparación, que en los últimos tiempos se ha incrementado considerablemente, lo que ha provocado que se preste mucha atención a aspectos físicos, técnicos, estilísticos y artísticos enfocados en su rendimiento (Sánchez, 2018). Para ello, la mayoría de los docentes siguen formándose de manera continua, con la idea de adquirir conocimientos concretos que les permitan mejorar su práctica docente. Debido al creciente interés por el máximo 


\section{Sánchez-Izquierdo et al.}

rendimiento de los bailarines y bailarinas de danza clásica, en los últimos años han aumentado las investigaciones encaminadas a analizar aspectos de la técnica de la danza que permitan mejorar el rendimiento (Mc Hugh y Cosgrave, 2010; Plaza, 2019; Wyon, 2010).

La Metodología Observacional (MO) es un procedimiento científico altamente flexible y riguroso que estudia las conductas perceptibles directa y/o indirectamente que ocurren preferentemente en contextos naturales (Anguera y Hernández-Mendo, 2013, 2014, 2015). Se puede considerar Mixed Method en sí misma, pues engloba una primera fase de carácter cualitativa que a partir de la obtención de parámetros procedentes de los registros observacionales se transforma en una fase cuantitativa (Anguera y Hernández-Mendo, 2014, 2016).

En la actualidad se convierte en un elemento de sostenibilidad metodológico en todos los ámbitos de aplicabilidad (Anguera y Hernández-Mendo, 2016). $\mathrm{Se}$ ha visto beneficiada por el desarrollo de los softwares y las tecnologías, como LINCE (Gabín, Camerino, Anguera y Castañer, 2012), THEMECODER (Borrie, Jonsson y Magnusson, 2001, 2002), MOTS (Castellano, Perea, Alday y Hernández-Mendo, 2008) y HOISAN (HernándezMendo, López-López, Castellano, Morales-Sánchez y Pastrana, 2012).

Su notable avance, y el de los programas informáticos, facilitan el diseño y validación de herramientas de observación ad hoc. En el deporte, su mayor utilización se registra en estudios de elementos técnicos, tácticos, metodológicos y estratégicos (Castañer, Torrents, Anguera y Dinušová, 2009; Hernández-Mendo, Díaz-Martínez y MoralesSánchez, 2010; Vázquez-Diz, Morillo-Baro, Reigal, Morales-Sánchez y Hernández-Mendo, 2019).

En los últimos años se han generado un gran número de investigaciones teóricas y aplicadas con el uso de la MO (Gabín et al., 2012; Pérez-Tejera, Valera y Anguera, 2018). Su mayor nivel de utilización se ha producido en el ámbito de la actividad física, fundamentalmente en estudios de educación física (Fernández, Sánchez, Jiménez, Navarro y Anguera, 2012; Santamaría, Ruiz, Puchalt, Ros y Martin, 2016) y el deporte (Anguera, Portell, Chacón-Moscoso y Sanduvete-Chaves, 2018; Jiménez-Salas Morillo-
Baro, Reigal, Morales-Sánchez y Hernández-Mendo, 2020; Menescardi, Falco, Ros, Morales-Sánchez y Hernández-Mendo, 2019); aunque también se evidencia su uso en las manifestaciones acrobáticas (Grau, 2018) y artísticas, donde se identifican y analizan respuestas motrices en la danza y en la expresión corporal (Castañer et al., 2009); y de instrumentos de observación ad hoc para el análisis de acciones motrices en la danza Contemporánea, Expresión Corporal y Danza Contact-Improvisation (Castañer et al., 2009).

La MO en los estudios sobre la danza podrá contribuir a la investigación científica en dicho campo. Su utilización ofrecerá: (1) La posibilidad de analizar la conducta de los bailarines tanto en situaciones reales como en sus entrenamientos, audiciones, competiciones, etc. ofreciendo información útil para su desarrollo. (2) Analizar ejercicios de Danza Clásica de otras especialidades de danza, como la Danza Española (Escuela Bolera, Folklore, Danza Estilizada), el Baile Flamenco, la Danza Contemporánea... Por ejemplo, codificando un ballet de repertorio de Escuela Bolera como el Zapateado de María Cristina o los Panaderos de la Tertulia; para el Baile Flamenco realizando un instrumento $a d$ hoc que codifique un palo concreto como las Soleares; para la Danza Estilizada codificando Andalucía (Lecuona, 1984) o Sevilla (Albéniz, 2006). (3) Estimar los patrones de conducta de los bailarines/as en los entrenamientos y su eficacia a través de patrones de éxito/fracaso en las competiciones o exámenes.

En danza clásica, no se ha encontrado evidencia de trabajos que presenten una herramienta de observación ad hoc compuesta por un sistema mixto de formato de campo y un sistema de categorías exhaustivas y mutuamente excluyentes (E/ME), con el objetivo de codificar algún ejercicio de danza clásica de barra o de centro, por lo que consideramos de interés su desarrollo por la aportación que podría suponer.

El objetivo de este estudio es construir y validar una herramienta de observación del ejercicio del plié en una barra de danza clásica que permita su registro y codificación; cumpliendo los criterios del análisis de la Calidad del Dato y análisis de Generalizabilidad. 


\section{Observación de la evaluación técnica en danza clásica}

\section{Diseño}

El diseño observacional utilizado en esta investigación se encuentra ubicado en el cuadrante I y es de carácter puntual/idiográfico/multidimensional (Anguera, Blanco-Villaseñor, Hernández-Mendo y Losada, 2011). El diseño incorpora un seguimiento intrasesional.

\section{Participantes}

El estudio está formado por 10 participantes, 8 bailarinas $(80 \%)$ y 2 bailarines $(20 \%)$ con certificaciones profesionales de danza clásica CIED CID UNESCO y con edades comprendidas entre 16 y 24 años. Todos han superado los estudios completos de Ballet Elementary y los tres niveles de Professional Ballet CIED CID UNESCO.

\section{Instrumentos}

Para la elaboración del sistema de codificación se utilizó una estrategia "empírico-inductiva", debido a la inexistencia de construcciones teóricas en la danza clásica. Se diseñó una herramienta de observación $a d$ hoc utilizando un sistema mixto de formato de campo y sistemas de categorías exhaustivas y mutuamente excluyentes (E/ME) (Anguera, 1979; HernándezMendo, 1996; Castellano, 2000) formado por 5 criterios y 84 categorías que se corresponden con todos los movimientos que integran la técnica del ejercicio del Plié objeto de estudio. Todos los criterios están construidos como sistemas de categorías. La tabla 1 presenta los criterios, categorías y sistema de codificación de la herramienta observacional.

Tabla 1. Criterios, categorías y códigos de la herramienta observacional evaluación técnica en la danza clásica: Ejercicio del plié

\begin{tabular}{|c|c|c|}
\hline Criterios & Códigos & Categorías \\
\hline \multirow{8}{*}{$\begin{array}{l}\text { 1. Tren } \\
\text { Inferior } \\
\text { (TI) }\end{array}$} & PBGP1 & $\begin{array}{l}\text { Principio de la bajada del } \\
\text { grand plié en } 1^{\text {a }} \text { posición. }\end{array}$ \\
\hline & 1POSTI & $1^{\mathrm{a}}$ posición. \\
\hline & 2POSTI & $2^{\mathrm{a}}$ posición. \\
\hline & PBGP2 & $\begin{array}{l}\text { Principio de la bajada del } \\
\text { grand plié en } 2^{\mathrm{a}} \text { posición. }\end{array}$ \\
\hline & RELEV2 & Relevé en $2^{\mathrm{a}}$ posición. \\
\hline & REV4DDV & $\begin{array}{l}\text { Relevé en } 4^{\mathrm{a}} \text { posición } \\
\text { derecha delante. }\end{array}$ \\
\hline & 4POSDDVTI & $\begin{array}{l}4^{\mathrm{a}} \text { posición derecha } \\
\text { delante. }\end{array}$ \\
\hline & PBGP4DDV & $\begin{array}{l}\text { Principio de la bajada del } \\
\text { grand plié en } 4^{\mathrm{a}} \text { derecha } \\
\text { delante. }\end{array}$ \\
\hline
\end{tabular}

5 RDDDACDP $\quad 5^{\mathrm{a}}$ Retiré derriére derecho detrás a la altura del coud de pied.

5 RDDDAR $\quad 5^{\mathrm{a}}$ retiré derriére derecho detrás a la altura de la rodilla.

5 RDDDEG $\quad 5^{\mathrm{a}}$ retiré derriére derecho detrás por encima del gemelo.

5 RDER

5POSIDTI

PBGP5IDV

ADRRDD90 ${ }^{\circ}$

BJMGP1

BJMGP2

BJMGP4DDV

BJMGP5IDV

RV5IDV

DPLIÉ1

DPLIÉ2

DPLIÉ5IDV

TDDV4

RV5DDV

RELV1

TEND2

TDD4SDP

PSGP1

PSGP4DDV

PSGP5IDV

PSGP2 $5^{\mathrm{a}}$ retiré derriére por encima de la rodilla.

$5^{\mathrm{a}}$ posición izquierda delante.

Principio de la bajada del grand plié en $5^{\mathrm{a}}$ posición izquierda delante.

Attitude derriére relevé derecho detrás $90^{a}$ o más.

Bajada máxima grand plié en $1^{\mathrm{a}}$.

Bajada máxima en $2^{a}$ posición sin subir los talones.

Bajada máxima del grand plié en $4^{\mathrm{a}}$ posición derecha devant.

Bajada máxima grand plié en $5^{\mathrm{a}}$ izquierda delante.

Relevé $5^{a}$ izquierda devant. Demi plié en $1^{\mathrm{a}}$ posición.

Demi plié $2^{\mathrm{a}}$ en segunda posición.

Demi plié $5^{\text {a }}$ izquierda delante.

Tendu derecha devant $4^{\mathrm{a}}$ posición.

Relevé en $5^{\text {a derecha }}$ delante.

Relevé en $1^{a}$ posición.

Tendu derecha a $2^{\mathrm{a}}$ posición.

Tendú derecha devant $4^{\mathrm{a}}$ saliendo de demi plié.

Principio de la subida del grand plié en $1^{\mathrm{a}}$ posición.

Principio de la subida del grand plié en $4^{\mathrm{a}}$ posición derecha delante.

Principio de la subida del grand plié en $5^{\mathrm{a} i z q u i e d a}$ delante.

Principio de la subida del grand plié en $2^{\mathrm{a}}$ posición. 


\section{Sánchez-Izquierdo et al.}

EADRDD90 Estirar Acttitude derrière relevé derecho detrás a $90^{\circ}$ o más.

\begin{tabular}{lll} 
& ERRTI & Error en el tren inferior. \\
\cline { 2 - 3 } 2. Tren & 1POSDTS & $\begin{array}{l}1^{\text {a }} \text { posición derecha. } \\
\text { Superior }\end{array}$ \\
$\begin{array}{ll}1^{\mathrm{a}} \text { posición izquierda. } \\
\text { (TS) }\end{array}$ & 5POSITS & $\begin{array}{l}5^{\mathrm{a}} \text { posición, brazo derecho } \\
\text { arriba. }\end{array}$
\end{tabular}

5POSITS $\quad 5^{\mathrm{a}}$ posición, brazo

2POSDTS $\quad 2^{\mathrm{a}}$ posición derecha.

2POSITS $\quad 2^{\mathrm{a}}$ posición izquierda.

2ALNGD $\quad 2^{\mathrm{a}}$ allongué derecho.

2ALNGI $\quad 2^{\text {a }}$ allongué izquierdo.

5ALNGD $\quad 5^{\text {a }}$ allongué derecha.

BRBASD Brazos en posición de preparatoria o bras bas derecha.

BRBASI Brazos en posición de preparatoria o bras bas izquierda.

PBSDV2AL Principio de la bajada al souplesse delante con brazos en segunda posición alargada.

BJMXSDV5 Bajada máxima al souplesse delante con brazos en $5^{\mathrm{a}}$ posición.

PSSDV5 Principio de la subida del souplesse delante con brazos en $5^{\text {a }}$ posición.

PCCTDBD5 Principio del cambré de coté dentro con brazo derecho en $5^{\mathrm{a}}$ posición.

MCCTBD5 Máximo cambré de coté dentro con brazo derecho en $5^{\mathrm{a}}$ posición.

MCDRI5 Máximo cambré derriére con brazo izquierdo en $5^{\text {a }}$ posición.

\begin{tabular}{|c|c|c|}
\hline \multirow{6}{*}{$\begin{array}{l}\text { 3. Cabeza/ } \\
\text { Mirada } \\
(\mathrm{C} / \mathrm{M})\end{array}$} & \multirow{2}{*}{$\begin{array}{l}\text { ERRTS } \\
1 / 8 \mathrm{PD}\end{array}$} & Error en el tren superior. \\
\hline & & $\begin{array}{l}1 / 8 \text { de perfil derecho, } \\
\text { mirada a la altura de los } \\
\text { ojos. }\end{array}$ \\
\hline & 1/8PI & $\begin{array}{l}1 / 8 \text { de perfil izquierdo, } \\
\text { mirada a la altura de los } \\
\text { ojos. }\end{array}$ \\
\hline & 1/8PIDDH & $\begin{array}{l}1 / 8 \text { de perfil con } \\
\text { inclinación derecha dehors. }\end{array}$ \\
\hline & 1/8PDMM2 & $\begin{array}{l}1 / 8 \text { de perfil derecha } \\
\text { mirada a la mano de la } 2^{\mathrm{a}} \\
\text { posición. }\end{array}$ \\
\hline & 1/8PDMM5 & $\begin{array}{l}1 / 8 \text { de perfil derecha } \\
\text { mirada a la mano de la } 5^{\text {a }} \\
\text { posición. }\end{array}$ \\
\hline
\end{tabular}

1/8PIDDHM2 1/8 de perfil inclinación derecha dehors mirando a la mano de la $2^{\text {a }}$ posición.

1/8PFIMM2 $1 / 8$ de perfil izquierda mirada a la mano de la $2^{\mathrm{a}}$ posición.

1/8PFIMM5 $1 / 8$ de perfil izquierda mirada a la mano de la $5^{\text {a }}$ posición.

ENFACE De frente, mirada al frente, a la altura de los ojos.

FIJA Cabeza fija, mirada a la altura de los ojos.

FLEX Cabeza en flexión mirada abajo.

1/8PIDDHMM5 1/8 de perfil inclinación derecha en dehors mirando a la mano de la $5^{\text {a }}$ posición.

IDDMM1 Inclinación derecha en dedans mirando a la mano de la $1^{\text {a }}$ posición.

IIDMM1 Inclinación izquierda en dedans mirando a la mano en $1^{\mathrm{a}}$.

IDDMMBS Inclinación derecha en dedans mirando a la mano que está en bras bas.

IIDMMBS Inclinación izquierda en dedans mirando a la mano que está en bras bas.

\begin{tabular}{|c|c|c|}
\hline & $\begin{array}{l}\text { PUNT2D } \\
\text { PUNT2I } \\
\text { ERRCM }\end{array}$ & $\begin{array}{l}\text { Punto } 2 \text { derecho. } \\
\text { Punto } 2^{\mathrm{a}} \text { izquierdo. } \\
\text { Error en cabeza/mirada. }\end{array}$ \\
\hline \multirow{5}{*}{$\begin{array}{l}4 . \\
\text { Dirección } \\
\text { espacial } \\
\text { (DE) }\end{array}$} & COTBDF & De coté a la barra lado \\
\hline & COTBIF & $\begin{array}{l}\text { derecho fuera. } \\
\text { De coté a la barra dentro } \\
\text { lado izquierdo fuera. }\end{array}$ \\
\hline & EFCAB & Effacé a la barra. \\
\hline & EFCFBD2 & $\begin{array}{l}\text { Effacé fuera de la barra, } \\
\text { diagonal } 2^{\mathrm{a}} \text {. }\end{array}$ \\
\hline & ERRDE & Error dirección espacial. \\
\hline 5. Cuentas & 1 & Cuenta en el tiempo 1. \\
\hline Musicales & 2 & Cuenta en el tiempo 2. \\
\hline (CC) & 3 & Cuenta en el tiempo 3. \\
\hline & 4 & Cuenta en el tiempo 4. \\
\hline & 5 & Cuenta en el tiempo 5. \\
\hline & 6 & Cuenta en el tiempo 6. \\
\hline & 7 & Cuenta en el tiempo 7. \\
\hline & 8 & Cuenta en el tiempo 8. \\
\hline & ERRCC & Error en cuenta musical. \\
\hline
\end{tabular}

El criterio tren inferior (TI) está compuesto por 33 categorías referidas a los movimientos que se ejecutan con la parte baja, conformada por las piernas y los 


\section{Observación de la evaluación técnica en danza clásica}

pies. La categoría 1POSTI, referida a la primera posición de pies, se marca cuando existe una correspondencia de dicha posición con el ejercicio marcado. Siempre que no exista correspondencia de los movimientos con la del modelo a seguir, aparecerá marcada la categoría ERRTI, que hace referencia a error en el tren inferior. La categoría BJMGP4DDV se marcará cuando, según la pauta marcada por el modelo a seguir, el participante deba encontrarse en su bajada máxima del grand plié en cuarta posición con la pierna derecha delante, manteniendo el correcto en dehors.

El criterio tren superior (TS) se refiere a la parte del tronco, brazos, manos, muñecas, dedos y tiene un total de 18 categorías. Los brazos son muy importantes en la danza clásica ya que complementan la línea y ayudan a los movimientos. La colocación de los brazos mantiene tres líneas: la interna que va desde la parte interna de la axila, el cúbito y el dedo meñique; la media que parte desde la mitad del hombro, codo y dedo corazón de la mano; la externa que recorre el hombro interno y llega al radio acabando en el dedo índice. En el estudio se han realizado posiciones de brazos como la $1^{\mathrm{a}}$, la $2^{\mathrm{a}}$ o la $5^{\mathrm{a}}$ posición, así como allongués, siendo estos últimos responsables de suavizar la línea y dar continuidad a los movimientos de los brazos. El estudio de las posiciones del tren superior, en concreto de los brazos, ayudarán a preparar y entrenar la coordinación de los movimientos que realizan los brazos como parte inherente a la técnica de la danza clásica y como su utilización independiente al movimiento de las piernas. Las manos tendrán una colocación concreta en donde el dedo pulgar se relaja hacia la primera articulación interfalángica del dedo corazón, sin llegar a tocarlo. El dedo índice sigue la línea externa del arco del brazo y el dedo anular y meñique se colocan en relación al dedo corazón. La categoría 2ALNGD se indica para señalar la segunda posición alargada de brazos. La categoría 5POSDTS indica que el brazo derecho del sujeto objeto de estudio está en quinta posición.

El criterio cabeza/mirada (CM) tiene un total de 19 categorías, las cuales se refieren a los movimientos que se realizan con la cabeza, así como las miradas que deben acompañar a dichos movimientos. Algunas de las posiciones son: 1/8PIDDHM2, que se refiere a 1/8 de perfil con inclinación derecha en dehors y mirada a la mano del brazo que está colocado en $2^{\mathrm{a}}$ posición;
ENFACE, si la cabeza está en posición de frente; FLEX, si se mantiene en flexión; ERRCM, referido al error de la categoría cabeza/mirada, si el participante no realiza el movimiento indicado según la secuencia anteriormente marcada.

El criterio dirección espacial (DE) se compone de 5 categorías, las cuales tienen que ver con la dirección del participante respecto a la barra. La categoría COTBDF es cuando el participante realiza el ejercicio de perfil o de coté a la barra estando su brazo derecho fuera de ésta. La categoría COTBIF la identificamos cuando el participante realiza los pasos concretos y movimientos de perfil o de coté a la barra estando su brazo izquierdo fuera. Marcaremos la categoría ERRDE, cuando no coincida la dirección espacial del participante respecto a la barra con el ejercicio propuesto.

El criterio cuentas musicales (CC) está formado por 9 categorías, las cuales se dividen en 8 tiempos musicales $(1,2,3,4,5,6,7$ y 8$)$ y un error (ERRCC). Cada movimiento debe cuadrar en una cuenta musical. La música es un recurso didáctico de enorme valor para la clase de danza clásica, pues proporciona la estructura que sirve de soporte a los movimientos que componen los ejercicios. La música establece el tiempo y el ritmo adecuado en el estudio del plié, ayudando a la realización de la diferente secuencia de movimientos, calidad y fluidez de los pliés dando un aspecto ligado, elástico, suave... de manera que ayude a la sensación de muelle, característica principal de dicho ejercicio. El fraseo es importante, utilizándose en este estudio frases de 8 compases, de forma que los ejercicios comienzan al empezar una frase y finalizan cuando ésta termina. El compás utilizado para el plié es un 4/4. La categoría cuenta musical 1 coincidirá con el movimiento musical pautado para la cuenta 1 , la categoría musical 2 para el movimiento montado en el tiempo 2, hasta los 8 tiempos, siendo la categoría ERRCC el error que se marcará cuando el participante no desarrolle los movimientos en el tiempo musical marcado.

A continuación, en la tabla 2 se muestra un ejemplo de definición de una categoría, donde se incluye el núcleo categorial y su nivel de plasticidad. 


\section{Sánchez-Izquierdo et al.}

Tabla 2. Definición de una categoría: núcleo categorial y nivel de plasticidad

\begin{tabular}{ll}
\hline DPLIÉ1 & $\begin{array}{l}\text { Demi-Plié en } 1^{\mathrm{a}} \text { posición. Media } \\
\text { flexión. }\end{array}$ \\
\hline Núcleo categorial & $\begin{array}{l}\text { Se realiza media flexión en } 1^{\mathrm{a}} \\
\text { posición. }\end{array}$ \\
\hline & $\begin{array}{l}\text { Se marcará cuando el participante } \\
\text { realice la media flexión en } 1^{\mathrm{a}}\end{array}$ \\
Nivel de & $\begin{array}{l}\text { posición sin levantar los talones, con } \\
\text { plasticidad o } \\
\text { grado de apertura }\end{array}$ \\
& $\begin{array}{l}\text { las rodillas encima del empeine y } \\
\text { manteniendo el en dehors en el } \\
\text { ejercicio. }\end{array}$ \\
\hline
\end{tabular}

\section{Procedimiento}

El trabajo fue aprobado por el Comité de Ética de la Universidad de Málaga $\mathrm{n}^{\circ}$ 19-2015-H. Además, se siguieron las directrices de la Declaración de Helsinki (2013) y se solicitó el Consentimiento Informado de los participantes, realizado de acuerdo a lo dispuesto en la Ley Orgánica 15/1999 de 13 de diciembre de Protección de Datos de Carácter Personal (LOPD). Este estudio cumple con las recomendaciones del Consejo General de Colegios de Psicólogos.

Las grabaciones se desarrollaron en una academia de danza. La docente experta en danza clásica explicó y demostró los ejercicios a los bailarines y bailarinas. Utilizó el método de reproducción de modelos, donde la propia profesional resolvía las posibles dudas. Después de realizar en varias ocasiones cada ejercicio se procedió a la grabación de cada participante. Posteriormente la directora del centro de Danza proporcionó la grabación de los videos para su visionado y análisis.

La unidad de análisis ha sido el plié en una barra de danza clásica, registrándose: el tren inferior, el tren superior, la cabeza/mirada, la dirección espacial y las cuentas musicales.

Se inicia con la preparación del ejercicio y la espera musical desde la cuenta 3, 5, 6, y la preparación en la cuenta 7 y 8 . El tren inferior comienza colocado en una primera posición, los brazos en bras bas, la cabeza mirando fuera de la barra al punto $2^{\circ}$ y la dirección espacial respecto a la barra, de lado a ésta, con pierna derecha fuera. Se registraron los ejercicios del plié en sus diferentes posiciones $1^{\circ}, 2^{\circ}, 4^{\mathrm{a}}$ y $5^{\mathrm{a}}$, realizándose esta última, con la pierna izquierda fuera de la barra.
El ejercicio finaliza en $5^{\mathrm{a}}$ posición de coté a la barra con la pierna izquierda fuera de ésta, los brazos en bras bas y la cabeza fuera de la barra a la diagonal izquierda.

Terminada la recogida de datos, el observador debe tener la garantía necesaria sobre su calidad (Anguera, 2003). Con la utilización del software HOISAN (Hernández-Mendo et al., 2012) se realizó el registro y codificación de las observaciones, así como el análisis de calidad del dato de carácter cuantitativo a partir de la estimación de los coeficientes de correlación de Pearson, Spearman y Tau-B de Kendall, y para el concepto de concordancia, el índice Kappa de Cohen.

Durante el proceso, el observador 1 registró todos los ejercicios del plié de los 10 participantes. Pasados quince días, volvió a registrar los mismos ejercicios del plié a los 10 participantes con el objetivo de determinar los resultados de la concordancia intraobservador.

Cuando el observador 2 mostró en los entrenamientos un alto dominio de la herramienta, procedió a registrar los ejercicios procedió a registrar los ejercicios del plié de los 10 participantes con el objetivo de determinar la concordancia interobservador.

Culminado este análisis, se procedió a realizar un Análisis de Generalizabilidad mediante el programa informático SAGT (Hernández-Mendo, BlancoVillaseñor, Pastrana, Morales-Sánchez, y RamosPérez, 2016; Hernández-Mendo, Ramos-Pérez y Pastrana, 2012). La Teoría de Generalizabilidad (Cardinet, Tourneur y Allal, 1976, 1981; Cronbach, Gleser, Nanda y Rajaratnam, 1972) unifica las definiciones de fiabilidad, validez y precisión (Blanco-Villaseñor et al., 2014) y comprende cuatro etapas: la definición de las facetas de estudio, el análisis de varianza de las puntuaciones obtenidas sobre las facetas de estudio, el cálculo de los componentes de error y la optimización de los coeficientes de Generalizabilidad (Blanco-Villaseñor et al., 2014). Con el objetivo de complementar el análisis de calidad del dato, se estimó el acuerdo intraobservador e interobservador, la homogeneidad de las categorías y el número mínimo de sesiones necesarias para generalizar con precisión. 


\section{Observación de la evaluación técnica en danza clásica}

\section{RESULTADOS}

Se presentan los resultados de todos los análisis llevados a cabo en el estudio.

Fiabilidad de la herramienta observacional

Desde el punto de vista cuantitativo se analiza la Calidad del Dato a partir de los coeficientes de correlación Pearson, Spearman y Tau b de Kendall, y el índice de concordancia Kappa de Cohen (tabla 3).

Tabla 3. Resultados de los coeficientes de correlación Pearson, Spearman y Tau b de Kendall, y el índice de concordancia Kappa de Cohen

\begin{tabular}{|c|c|c|c|c|}
\hline \multicolumn{5}{|c|}{ Coeficientes de Correlación } \\
\hline Coeficiente & $\begin{array}{l}\text { Conco } \\
\text { Observ }\end{array}$ & $\begin{array}{l}\text { ancia Intra } \\
\text { dor }\end{array}$ & $\begin{array}{l}\text { Concordan } \\
\text { Observado }\end{array}$ & ia Inter \\
\hline Participantes & $1,2,4,9$ & $3,5,6,7,8,10$ & $2,3,4,5,6,9$ & $1,7,8,10$ \\
\hline Pearson & .99 & 1.00 & .99 & 1.00 \\
\hline Spearman & .99 & 1.00 & .99 & 1.00 \\
\hline $\begin{array}{l}\text { Tau b de } \\
\text { Kendall }\end{array}$ & .99 & 1.00 & .99 & 1.00 \\
\hline \multicolumn{5}{|c|}{ Índice de Concordancia } \\
\hline $\begin{array}{l}\text { Kappa de } \\
\text { Cohen }\end{array}$ & .99 & 1.00 & .99 & 1.00 \\
\hline
\end{tabular}

Los resultados que se muestran en la tabla 3 se consideran resultados adecuados para la obtención de registros fiables.

A continuación, la tabla 4 muestra los resultados del cálculo del índice de concordancia Kappa de Cohen, con valores siempre superiores a 0.96 .

Tabla 4. Calidad del Dato I.

\begin{tabular}{lcccccc}
\hline Participantes & \multicolumn{3}{c}{ Kappa Cohen } & \multicolumn{3}{c}{ Kappa Cohen } \\
& \multicolumn{3}{c}{ Intra } & & \multicolumn{3}{c}{ Inter } \\
\hline $\begin{array}{l}\text { Sesiones de } \\
\text { observación }\end{array}$ & $1-2$ & $1-3$ & $2-3$ & $1-2$ & $1-3$ & $2-3$ \\
\hline Participante 1 & 1.00 & 1.00 & 1.00 & 1.00 & 1.00 & 1.00 \\
Participante 2 & 0.99 & 0.99 & 1.00 & 0.99 & 0.98 & 1.00 \\
Participante 3 & 1.00 & 1.00 & 1.00 & 0.98 & 1.00 & 0.98 \\
Participante 4 & 1.00 & 1.00 & 1.00 & 1.00 & 1.00 & 1.00 \\
Participante 5 & 1.00 & 1.00 & 1.00 & 1.00 & 1.00 & 1.00 \\
Participante 6 & 1.00 & 1.00 & 1.00 & 0.98 & 0.99 & 1.00 \\
Participante 7 & 1.00 & 1.00 & 1.00 & 1.00 & 1.00 & 1.00 \\
Participante 8 & 1.00 & 1.00 & 1.00 & 1.00 & 1.00 & 1.00 \\
Participante 9 & 0.99 & 0.99 & 1.00 & 0.96 & 0.98 & 1.00 \\
Participante 10 & 1.00 & 1.00 & 1.00 & 1.00 & 1.00 & 1.00 \\
\hline
\end{tabular}

La tabla 5 muestra los resultados del cálculo del índice de concordancia canónica de Krippendorf.

Tabla 5. Calidad del Dato II

\begin{tabular}{lcc}
\hline Participantes & $\begin{array}{c}\text { Concordancia } \\
\text { Canónica } \\
\text { Intra }\end{array}$ & $\begin{array}{c}\text { Concordancia } \\
\text { Canónica } \\
\text { Inter }\end{array}$ \\
\hline Participante 1 & $100 \%$ & $100 \%$ \\
Participante 2 & $99.61 \%$ & $99.61 \%$ \\
Participante 3 & $100 \%$ & $98.66 \%$ \\
Participante 4 & $100 \%$ & $100 \%$ \\
Participante 5 & $100 \%$ & $100 \%$ \\
Participante 6 & $100 \%$ & $99.61 \%$ \\
Participante 7 & $100 \%$ & $100 \%$ \\
Participante 8 & $100 \%$ & $100 \%$ \\
Participante 9 & $99.61 \%$ & $98.83 \%$ \\
Participante 10 & $100 \%$ & $100 \%$ \\
\hline
\end{tabular}

\section{Análisis de Generalizabilidad}

La Teoría de la Generalizabilidad (Cardinet et al., 1981; Cronbach at al., 1979) permite que se controle la adecuada calidad de los datos que proceden de la MO y posibilita establecer una estimación precisa de los diferentes tamaños muestrales.

El análisis de Generalizabilidad (Blanco-Villaseñor et al., 2014) ha determinado la fiabilidad entre los observadores (acuerdo interobservador) utilizando un diseño de dos facetas (categorías y observadores= $\mathrm{CA} / \mathrm{O}$ ). Los datos obtenidos mediante el programa informático SAGT declara un porcentaje alto de varianza asociado a la faceta categorías (99.92\%), siendo 0 para la faceta de observadores y de $0.07 \%$ en la interacción de las facetas categorías/observadores. El coeficiente de generalizabilidad relativo (fiabilidad) es de 1.00 y el coeficiente de generalizabilidad absoluto (generalizabilidad) es 1.00, por lo que los resultados son excelentes.

Para determinar la fiabilidad intraobservador se ha tomado el mismo diseño. Los resultados indican una varianza asociada a la faceta categorías de $99.97 \%$, siendo 0 para la faceta observadores y de $0.03 \%$ para la interacción de las facetas categorías/observadores. El coeficiente de generalizabilidad relativo es $1.00 \mathrm{y}$ el coeficiente de generalizabilidad absoluto es 1.00 . Resultados igualmente excelentes. 


\section{Sánchez-Izquierdo et al.}

Para estimar la homogeneidad de las categorías, se ha utilizado un diseño cruzado de dos facetas, observadores y categorías $=\mathrm{O} / \mathrm{CA}$, comprobando el grado de diferenciación de las distintas partes del plié utilizando las categorías propuestas. Los coeficientes de generalizabilidad para esta estructura de diseño son nulos (0.00 y 0.00); indicando que la homogeneidad de las categorías es óptima, en el sentido de diferenciadora (Blanco-Villaseñor, et al., 2014).

Para la estimación del número mínimo de sesiones que se necesitan para generalizar con precisión, se ha utilizado un diseño de dos facetas, categorías y sesiones (CA/S). La estimación de los componentes de varianza se ha llevado a cabo de forma aleatoria infinita para las categorías y las sesiones. El análisis muestra que una alta variabilidad queda asociada a la faceta categorías $(99.91 \%)$, siendo nula para la faceta sesiones $(0.00 \%)$ y quedando el resto de la variabilidad para la faceta de interacción categorías/sesiones en $0.08 \%$. Con esta estructura de diseño, se puede determinar que con la observación de una sesión se alcanzaría un óptimo resultado con un coeficiente de generalizabilidad relativo de $1.00 \mathrm{y}$ un coeficiente absoluto de 1.00 .

Tabla 6. Resultados del Análisis de Generalizabilidad

\begin{tabular}{cccc}
\hline $\begin{array}{c}\text { Análisis de } \\
\text { Generalizabilidad }\end{array}$ & Facetas & $\begin{array}{c}\text { Coeficiente } \\
\text { G } \\
\text { Relativo }\end{array}$ & $\begin{array}{c}\text { Coeficiente } \\
\text { G } \\
\text { Absoluto }\end{array}$ \\
\hline $\begin{array}{c}\text { Fiabilidad } \\
\text { Interobservador } \\
\text { CA } / \mathrm{O}\end{array}$ & $\begin{array}{c}\mathrm{CA}=99.92 \% \\
\mathrm{O}=0 \%\end{array}$ & 1.00 & 1.00 \\
$\mathrm{C} / \mathrm{O}=0.07 \%$ & & \\
\hline $\begin{array}{c}\text { Fiabilidad } \\
\text { Intraobservador } \\
\text { CA/O }\end{array}$ & $\begin{array}{c}\mathrm{CA}=99.97 \% \\
\mathrm{O}=0 \%\end{array}$ & 1.00 & 1.00 \\
$\mathrm{C} / \mathrm{O}=0.03 \%$ & & \\
\hline Homogeneidad & $\mathrm{CA}=100 \%$ & 0.00 & 0.00 \\
$\mathrm{O} / \mathrm{CA}$ & $\mathrm{O}=0 \%$ & & \\
\hline Número de & $\mathrm{CA}=09 \%$ & & \\
Sesiones & $\mathrm{S}=0 \%$ & $1.09 \%$ & 1.00 \\
CA/S & $\mathrm{CA} / \mathrm{S}=0.08 \%$ & \multicolumn{2}{c}{1 Observación } \\
\hline
\end{tabular}

\section{DISCUSIÓN}

El objetivo del presente estudio era doble, por un lado, construir una herramienta de observación diseñada para codificar el ejercicio del plié en una barra de danza clásica, cumpliendo los criterios de fiabilidad, validez y precisión; y por otro, se pretendía analizar la
Generalizabilidad que permitiese determinar la fiabilidad de los observadores, estimar la bondad de las categorías y el número mínimo de sesiones necesarias para generalizar con precisión.

Los resultados obtenidos tras el análisis de la calidad del dato así como los de generalizabilidad han sido excelentes. Con el presente estudio se ha diseñado y validado la primera herramienta de observación $a d$ hoc para el análisis del ejercicio del plié en una barra de danza clásica. El procedimiento y el análisis realizado han sido satisfactorios al igual que en otras investigaciones en metodología observacional para el diseño y validación de herramientas ad hoc, como por ejemplo en el balonmano para el estudio del ataque posicional (Quiñones et al., 2019). Los datos confirman que la herramienta de observación diseñada permite registrar de manera fiable, precisa y válida el ejercicio del plié de una barra de danza clásica.

Aunque no se encuentran herramientas creadas previamente para el ejercicio del plié en una barra de danza clásica, existen instrumentos en ámbitos del deporte que evalúan los gestos técnicos de los deportistas, como en baloncesto (Garzón, Lapresa, Anguera y Arana, 2011) o atletismo (Lapresa, Solano, Arana, Anguera y Aragón, 2018); en ellos se hace uso de categorías que tienen en cuenta los tipos de acciones motoras del deportista; por ejemplo, cuando el portero de balonmano se encuentra en espagat, con una apertura de piernas alineadas y extendidas en dirección contraria (Vázquez-Diz et al., 2019).

Este estudio ha permitido, desde una perspectiva analítica, codificar el plié de una barra de danza clásica, atendiendo a sus diferentes partes y recogiendo los criterios que se necesitan para poder analizar los movimientos que conforman dicho ejercicio. Con la intención de alcanzar el grado de exhaustividad y exclusividad en cada sistemas de categorías, las conductas se agruparon en 5 criterios: tren inferior (TI), tren superior (TS), cabeza-mirada (CM), dirección espacial (DE) y cuentas musicales (CC).

La herramienta debía recoger todos estos criterios y categorías, pues en lo que a la técnica del plié se refiere, es uno de los ejercicios más importantes de la danza académica, ya que de éste depende que la danza se aprecie fluida, siendo de los primeros que coordina los diferentes movimientos que apreciamos en los 


\section{Observación de la evaluación técnica en danza clásica}

criterios estudiados: tren inferior, tren superior, cabeza-mirada, espacio y musicalidad. Entre las funciones del criterio tren inferior (TI) destaca la de servir de ayuda a desarrollar la elasticidad y la fuerza de las piernas de los bailarines y a trabajar el en dehors, entrenando la rotación externa de la articulación coxo-femoral y de los músculos rotadores. Permite desarrollar los ligamentos y articulaciones de las piernas y que la espalda adquiera fuerza para mantenerse recta. Su trabajo continuado favorece a diferentes contenidos de la técnica de la danza clásica, otorgando, por ejemplo a los saltos, el ballon, motivo por el que se debe insistir a los bailarines que liguen el movimiento y mantengan la rotación externa impidiendo que la energía propulsora de los músculos de las piernas se debiliten (González y Rodríguez, 2004).

En cuanto a la estructura del plié observado, desde sus comienzos se muestra claro y conciso, de manera que cada parte corporal muestra una posición definida desde el principio hasta el final del ejercicio, diferenciándose claramente los movimientos de las piernas (TI), el tronco y los brazos (TS), la cabezamirada (CM), la dirección espacial (DE) y las cuentas musicales (CC).

El entrenamiento riguroso de los observadores permitió obtener unos óptimos valores en los índices de correlación y en el análisis de Generalizabilidad; sin embargo, durante el desarrollo de este estudio se encontraron dificultades propias de la disciplina de la danza clásica, como la elevada cantidad de información a registrar.

Con todo, los resultados muestran que la herramienta de observación ad hoc diseñada recoge los criterios necesarios para codificar el ejercicio del plié en una barra de ballet y asegura una elevada validez, precisión y fiabilidad, lo que indica una alta calidad de los datos recogidos a través de dicho instrumento. Motivo por el que podrá suponer un avance en el campo científico de la danza y de las demás artes escénicas que requieran esta manera de codificar, abriendo caminos a que otras herramientas puedan ser validadas al igual que ésta.

La MO, la cual viene demostrando su gran utilidad en disciplinas deportivas (Anguera y Hernández Mendo, 2013 ; 2014) se manifiesta como la más idónea para ser utilizada en la evaluación técnica de la danza y de las artes escénicas en su contexto habitual, cuyo fin sea la optimización del rendimiento de los bailarines y bailarinas.

\section{APLICACIONES PRÁCTICAS}

En referencia a los resultados obtenidos en esta investigación, los datos generados aseguran posibles aplicaciones prácticas de dicha herramienta de observación ad hoc para el análisis del ejercicio del plié en una barra de ballet en diferentes grupos de bailarines, permitiéndonos apreciar de manera objetiva la ejecución precisa de los diferentes pasos propuestos, los errores que cometen en su ejecución, la precisión del ejercicio atendiendo a sus diferentes criterios, etc. sirviendo la creación de dicha herramienta como instrumento de observación y de evaluación del ejercicio del plié de una barra de danza clásica.

La presente investigación, pionera en la danza clásica, puede abrir caminos a la creación de otras herramientas de observación ad hoc para ser igualmente validadas. Y podría ayudar a los docentes que precisen de esta herramienta dentro del campo de la danza y/o de las artes escénicas en general. Su construcción puede permitir planificar y preparar los ejercicios utilizando toda la información para mejorar el rendimiento técnico de los bailarines/as.

\section{REFERENCIAS}

1. Albéniz, I. (2006). Sevilla (Sevillanas), Suite Española Op.47 No.3 para Guitarra (Sheet) (Español). Madrid: Real Musical; Edición: Real Musical.

2. Anguera, M. T. (1979). Observación de la conducta espacial. Comunicación presentada al VI Congreso Nacional de Psicología, Pamplona, España.

3. Anguera, M.T. (2003). La observación. En C. Moreno Rosset (Ed.), Evaluación psicológica. Concepto, proceso y aplicación en las áreas del desarrollo y de la inteligencia (pp. 271-308). Madrid: Sanz y Torres.

4. Anguera, M. T., Blanco, A., Hernández-Mendo, A. y Losada, J. L. (2011). Diseños observacionales: ajuste y aplicación en psicología del deporte. Cuadernos de Psicología del Deporte, 11(2), 63-76. https://doi.org/10.4321/s1578-84232015000100002

5. Anguera, M. T. y Hernández-Mendo, A. (2013). La metodología observacional en el ámbito del deporte. E-balonmano.com: Revista de Ciencias del Deporte 


\section{Sánchez-Izquierdo et al.}

9(3), 135-160. http://www.ebalonmano.com/ojs/index.php/revista/index

6. Anguera, M. T. y Hernández-Mendo, A. (2014). Metodología observacional y psicología del deporte: Estado de la cuestión. Revista de Psicología del Deporte, 23(1), 103-109. http://hdl.handle.net/2445/148502

7. Anguera, M. T. y Hernández-Mendo, A. (2015). Técnicas de análisis en estudios observacionales en ciencias del deporte. Cuadernos de Psicología del Deporte, 15(1), 13-30. https://doi.org/10.4321/S157884232015000100002

8. Anguera, M. T. y Hernández-Mendo, A. (2016). Avances en estudios observacionales de ciencias del deporte desde los mixed methods. Cuadernos de Psicología del Deporte, 16(1), 17-30. https://doi.org/10.4321/s1578-84232015000100002

9. Anguera, M. T., Portell, M., Chacón-Moscoso, S. y Sanduvete-Chaves, S. (2018). Indirect observation in everyday contexts: Concepts and methodological guidelines within a mixed methods framework. Frontiers in Psychology, 9:13. https://doi.org/10.3389/fpsyg.2018.00013

10. Asociación Médica Mundial (AMM) (2013). Declaración de Helsinki de la AMM - Principios éticos para las investigaciones médicas en seres humanos. 64 ${ }^{\mathrm{a}}$ Asamblea General; 2013 octubre. Fortaleza, Brasil: AMM. http://www.wma.net/es/30publications/10policies/b3/ https://doi.org/10.17126/joralres.2013.009

11. Blanco-Villaseñor, A., Castellano, J., HernándezMendo, A., Sánchez-López, C. R. y Usabiaga, O. (2014). Aplicación de la TG en el deporte para el estudio de la fiabilidad, validez y estimación de la muestra. Revista de Psicología del Deporte, 23(1), 131-137. https://ddd.uab.cat/record/11908

12. Borrie, A., Jonsson, G. K. y Magnusson, M. S. (2001). Application of T-pattern detection and analysis in sports research. Metodología de las Ciencias del Comportamiento, 3(2), 215-226. https://www.researchgate.net/publication/267270918

13. Borrie, A., Jonsson, G. K. y Magnusson, M. S. (2002). Temporal pattern analysis and its applicability in sport: An explanation and exemplar data. Journal of Sports Sciences, 20, 845-852. https://doi.org/10.1080/026404102320675675
14. Cardinet, J. Tourneur, Y. y Allal, L. (1976). The simmetry of generalizability theory: Applications to educational measurement. Journal of Educational Measurement, $13(2)$,

119-135. https://doi.org/10.1111/j.17453984.1976.tb00003.x

15. Cardinet, J. Tourneur, Y. y Allal, L. (1981). Extension of generalizability theory and its applications in educational measurement. Journal of Educational Measurement, 18(4), 183-204. https://doi.org/10.1111/j.17453984.1981.tb00852.x

16. Castañer, M., Torrents, C., Anguera, M. T. y Dinušová, M. (2009). Instrumentos de observación ad hoc para el análisis de las acciones motrices en Danza Contemporánea, Expresión Corporal y Danza Contact-Improvisation. Apunts, 95, 14-23. http://hdl.handle.net/10459.1/65383

17. Castellano, J. (2000). Observación y análisis de patrones de juego en el fútbol. Tesis Doctoral. Vitoria: Universidad del País Vasco.

18. Castellano, J., Perea, A., Alday, L. y HernándezMendo, A. (2008). The Measuring and Observation Tool in Sports. Behavior Research Methods, 40(3), 898-905. https://doi.org/10.3758/brm.40.3.898

19. Cronbach, L. J., Gleser, G. C., Nanda, H. y Rajaratnam, N. (1972). The dependability of behavioral measurements: theory of generalizability for scores and profiles. New York: John Wiley and Sons. https://doi.org/10.3102/00028312011001054

20. Fernández, M., Sánchez, C. R., Jiménez, F., Navarro, V. y Anguera, M. T. (2012). Sistema de codificación y análisis de la calidad del dato para una intervención inclusiva en Educación Física. Revista de Psicología del Deporte, 21(1), 67-73. http://hdl.handle.net/11181/4395.

https://doi.org/10.20868/upm.thesis.39683

21. Gabín, B., Camerino, O., Anguera, M. T. y Castañer, M. (2012). Lince: multiplatform sport analysis software. Procedia-Social and Behavioral Sciences, 46, 4692-4694. https://doi.org/10.1016/j.sbspro.2012.06.320

22. Garzón, B., Lapresa, D., Anguera, M. T. y Arana, J. (2011). Análisis observacional del lanzamiento de tiro libre en jugadores de baloncesto base. Psicothema, 23(4), 851-857.

23. González, M. C. y Rodríguez, G. (2004). Bases Metodológicas Referenciales. Técnica de la Danza 


\section{Observación de la evaluación técnica en danza clásica}

Clásica. Nivel Elemental. México: Consejo Nacional para la Cultura y las Artes.

24. Grau Torá, R. (2018). La Comunicación de las emociones a través del movimiento gimnástico. (Tesis doctoral). Universitat de Barcelona, España. Recuperado de: http://diposit.ub.edu/dspace/handle/2445/132248

25. Greene-Hass, J. (2010). Anatomía de la Danza. Guía Ilustrada para mejorar la flexibilidad, la fuerza y el tono muscular. Madrid: Ediciones Tutor.

26. Haskell, A. (1973). ¿Qué es el Ballet? Cuba: Cuadernos Populares.

27. Hernández-Mendo, A. (1996). Observación y análisis de patrones de juego en deportes sociomotores. (Tesis Doctoral sin publicar). Universidad de Santiago de Compostela, Santiago de Compostela, https://doi.org/10.14349/rlp.2019.v51.n3.3

28. Hernández-Mendo, A., Blanco-Villaseñor, A., Pastrana, J. L., Morales-Sánchez, V., Ramos-Pérez, F. J. (2016). SAGT: Aplicación informática para análisis de generalizabilidad. Revista Iberoamericana de Psicología del Ejercicio y el Deporte, 11(1), 77-89.

29. Hernández-Mendo, A., Díaz-Martínez, F. y Morales-Sánchez (2010). Construcción de una herramienta observacional para evaluar las conductas prosociales en las clases de educación física. Revista de Psicología del Deporte, 19(2), 305-318. https://doi.org/10.1344/did.2020.7.86-102

30. Hernández-Mendo, A., López López, J. A., Castellano, J., Morales-Sánchez, V. y Pastrana, J. L. (2012). Hoisan 1.2: Programa informático para uso en metodología observacional. Cuadernos de Psicología del Deporte, 12(1), 55-78. https://doi.org/10.4321/s1578-84232012000100006

31. Hernández-Mendo, A, Ramos-Pérez, F. y Pastrana, J. L. (2012). SAGT: Programa informático para análisis de Teoría de la Generalizabilidad. SAFE CREATIVE Código: 1204191501059.

32. Jiménez-Salas, J., Morillo-Baro, J. P., Reigal, R. E., Morales-Sánchez, V. y Hernández-Mendo, A. (2020). Polar coordinate analysis to study counterattacks in senior and under-16 men's handball. Cuadernos de Psicología del Deporte, 20(1), 48-61. https://doi.org/10.6018/cpd.396521
33. Lapresa, D., Solano, R., Arana, J., Anguera, M.T. y Aragón, S. (2018). Estudio observacional de la salida de tacos de atletismo en las fases específicas "a sus puestos" y "listos". Revista Iberoamericana de Psicología del Ejercicio y el Deporte, 13(1), 145-153.

34. Lecuona, E. (1984). Andalucía. "Suite Espagnole" for Piano. New York: Edward B Marks Music co.

35. McHugh, M. y Cosgrave C. (2010). To strech or not strech: the role os streching in injuty prevention and performance. Scandinavian Journal of Medicine y Science in sports, 20(1), 169-181. https://doi.org/10.1111/j.1600-0838.2009.01058.

36. Menescardi, C., Falco, C., Ros, C., MoralesSánchez, V. y Hernández-Mendo, A. (2019). Development of a taekwondo combat model based on Markov analysis. Front. Psychol. doi: https://doi.org/10.3389/fpsyg.2019.02188

37. Pérez-Tejera, F., Valera, S. y Anguera, M. T. (2018) Using Systematic Observation and Polar Coordinates Analysis to Assess Gender-Based Differences in Park Use in Barcelona. Front. Psychol. 9:2299. https://doi.org/10.3389/fpsyg.2018.02299

38. Plaza, E. P. (2019). Apuntes para la mejora de la flexibilidad en bailarines. Revista del Centro de Investigación Flamenco Telethusa, 12(14), 23 -29.

39. Quiñones Y., Morillo-Baro, J. P., Reigal, R. E., Morales-Sánchez, V., Vázquez-Diz, J. A. y Hernández-Mendo, A. (2019). El ataque posicional en balonmano: validación de un sistema de observación. Cuadernos De Psicología Del Deporte, 19(3), 113124. https://doi.org/10.6018/cpd.384091

40. Sánchez, M. (2018). Eutonía y Danza. La Investigación en Danza. Sevilla.11-115.

41. Santamaría, R., Ruiz, L., Puchalt, J. M., Ros, C. y Martin, J. (2016). Inclusión en las Aulas de Educación Física. Estudio de Casos. Sportis Sci J, 2(3), 496-514. https://doi.org/10.17979/sportis.2016.2.3.1511

42. Taccone, V. (2016). El ballet clásico. Observaciones sobre la técnica la disciplina y las influencias sobre el cuerpo del bailarín. IX Jornadas de Sociología de la UNLP, 5- 7 de diciembre. Argentina. En Memoria Académica. https://doi.org/10.14361/9783839448908-006

43. Vázquez-Diz, J. A., Morillo-Baro J. P., Reigal, R. E., Morales-Sánchez, V. y Hernández-Mendo, A. (2019). Mixed Methods in Decision-Making Through 


\section{Sánchez-Izquierdo et al.}

Polar Coordinate Technique: Differences by Gender on Beach Handball Specialist. Front. Psychol. 10: 1627. https://doi.org/10.3389/fpsyg.2019.01627
44. Wyon, M. (2010). Streching for Dance.International Association for Dance medicine y Sciencie. Bulleting for Teachers, 2(1), 9-11. 